\title{
RANDOM SUMMABILITY AND FOURIER SERIES
}

\author{
RICHARD BELLMAN
}

Fejér's theorem that the first arithmetic mean of the partial sums of the Fourier series of a function converges to the function p.p. is equivalent to saying the average values of the partial sums, taken in order, are close to the function. It is interesting to ask whether, if the partial sums are chosen at random, and then averaged, the new averages will be close to the function. We shall show that this is true most of the time.

Let us define random summability in terms of the existence of the limit

$$
\lim _{n \rightarrow \infty} \frac{\sum_{1}^{n} s_{k}(x)\left(1+r_{k}(t)\right)}{\sum_{1}^{n}\left(1+r_{k}(t)\right)}
$$

where $r_{k}(t)$ are the Rademacher functions, $r_{k}(t)=\operatorname{sign} \sin 2^{k+1} \pi t$, and $s_{k}(x)$ are the partial sums of $\sigma(f)=a_{0}+\sum_{1}^{\infty}\left(a_{k} \cos k x+b_{k} \sin k x\right)$.

The only fact we require concerning the Rademacher functions is $[1]^{1}:$ If $\sum_{1}^{\infty} a_{k}^{2}<\infty, \sum_{1}^{\infty} a_{k} r_{k}(t)$ converges p.p. in $t$.

Now we have

$$
\begin{aligned}
\frac{\sum_{1}^{n} s_{k}(x)\left(1+r_{k}(t)\right)}{\sum_{1}^{n}\left(1+r_{k}(t)\right)}= & \frac{\sum_{1}^{n} s_{k}(x)}{n} \cdot \frac{n}{\sum_{1}^{n}\left(1+r_{k}(t)\right)} \\
& +\frac{\sum_{1}^{n} s_{k}(x) r_{k}(t)}{n} \cdot \frac{n}{\sum_{1}^{n}\left(1+r_{k}(t)\right)} .
\end{aligned}
$$

Also $\sum_{1}^{\infty} r_{k}(t) / k^{1 / 2} \log (k+1)$ converges p.p. in $t$, by the above, since $\sum_{1}^{\infty} 1 / k \log (k+1)^{2}<\infty$, so that $\sum_{1}^{n} r_{k}(t)=o\left(n^{1 / 2} \log n\right)$, by Kronecker's Lemma (much more is known, but this is more than needed). Hence, certainly $\sum_{1}^{n} r_{k}(t)=o(n)$, and $\sum_{1}^{n}\left(1+r_{k}(t)\right) \sim n$.

By Fejér's theorem $n^{-1} \sum_{1}^{n} s_{k}(x) \rightarrow f(x)$ p.p. in $x$, so that it remains to prove $n^{-1} \sum_{1}^{n} s_{k}(x) r_{k}(t) \rightarrow 0$ p.p. in $x$ and $t$.

But $\sum_{1}^{\infty}\left(s_{k}(x) / k\right)^{2}<\infty$ p.p. in $x$, since $s_{k}(x)=o(\log k)$ p.p. in $x$ [2]. Therefore $\sum_{1}^{\infty} s_{k}(x) r_{k}(t) / k$ converges p.p. in $x$ and $t$, and using Kronecker's Lemma again $\sum_{1}^{n} s_{k}(x) r_{k}(t)=o(n)$.

Thus, we have proved: If $f(x)$ belongs to $L$, the Fourier series of $f(x)$ is random summable p.p. in $x$, most of the time.

Received by the editors February 16, 1943.

${ }^{1}$ Numbers in brackets indicate references at end of paper. 


\section{REFERENCES}

1. Zygmund, Trigonometrical series, chap. 5, p. 123.

2. Ibid., chap. 2, p. 32 .

UNIVERSITY OF WISCONSIN

\section{ON FIBRE SPACES. II}

RALPH H. FOX

This paper is primarily concerned with fibre mappings ${ }^{1}$ into an absolute neighborhood retract. Theorem ${ }^{2} 3$ is a converse of the covering homotopy theorem; it characterizes fibre mappings (into a compact ANR) as mappings for which the covering homotopy theorem holds. Theorem 4 is Borsuk's fibre theorem; ${ }^{3}$ the proof ${ }^{4}$ which I present here is new. It seems to me that this theorem is a promising tool in function-space theory. Also I think that it furnishes conclusive justification for the generality of the Hurewicz-Steenrod definition of a fibre space. In fact, a fibre space of the type constructed by Borsuk's theorem almost never has a compact base space and almost never has its fibres of the same topological type.

The common denominator of the proofs of Theorems 3 and 4 is a property which I call local equiconnectivity. Local equiconnectivity is a strengthened form of local contractibility and a weakened form of the absolute neighborhood retract property (Theorems 1 and 2). Definitions and notations are those of FS. I. ${ }^{5}$

Let $\Delta$ be the diagonal subset $\sum_{b \in B}(b, b)$ of $B \times B$. I shall call the space $B$ locally equiconnected (or, to be specific, $(U, V)$-equiconnected) if there are neighborhoods $U$ and $V$ of $\Delta$ and a homotopy $\lambda$ in $B$ between the two projections of $U$ which does not move the points of $\Delta$ and which is uniform ${ }^{5}$ with respect to $V$. Precisely:

(1) $\lambda_{t}\left(b_{0}, b_{1}\right)$ is defined for all $\left(b_{0}, b_{1}\right) \in U$,

(2) $\lambda_{0}\left(b_{0}, b_{1}\right)=b_{0}$,

Received by the editors April 2, 1943.

${ }^{1}$ W. Hurewicz and N. Steenrod, Proc. Nat. Acad. Sci. U. S. A. vol, 27 (1941) p. 61.

2 This theorem was announced in Hurewicz-Steenrod, op. cit. footnote 3.

${ }^{3}$ K. Borsuk, Fund. Math. vol. 28 (1937) p. 99.

4 This proof was announced in the author's paper On the deformation retraction of some function spaces $\cdots$, Ann. of Math. vol. 44 (1943) p. 52.

$5 \bar{\pi}(x, b)=(\pi(x), b)$ as in R. H. Fox, On fibre spaces. I, Bull. Amer. Math. Soc. vol. 49 (1943) pp. 555-557. 